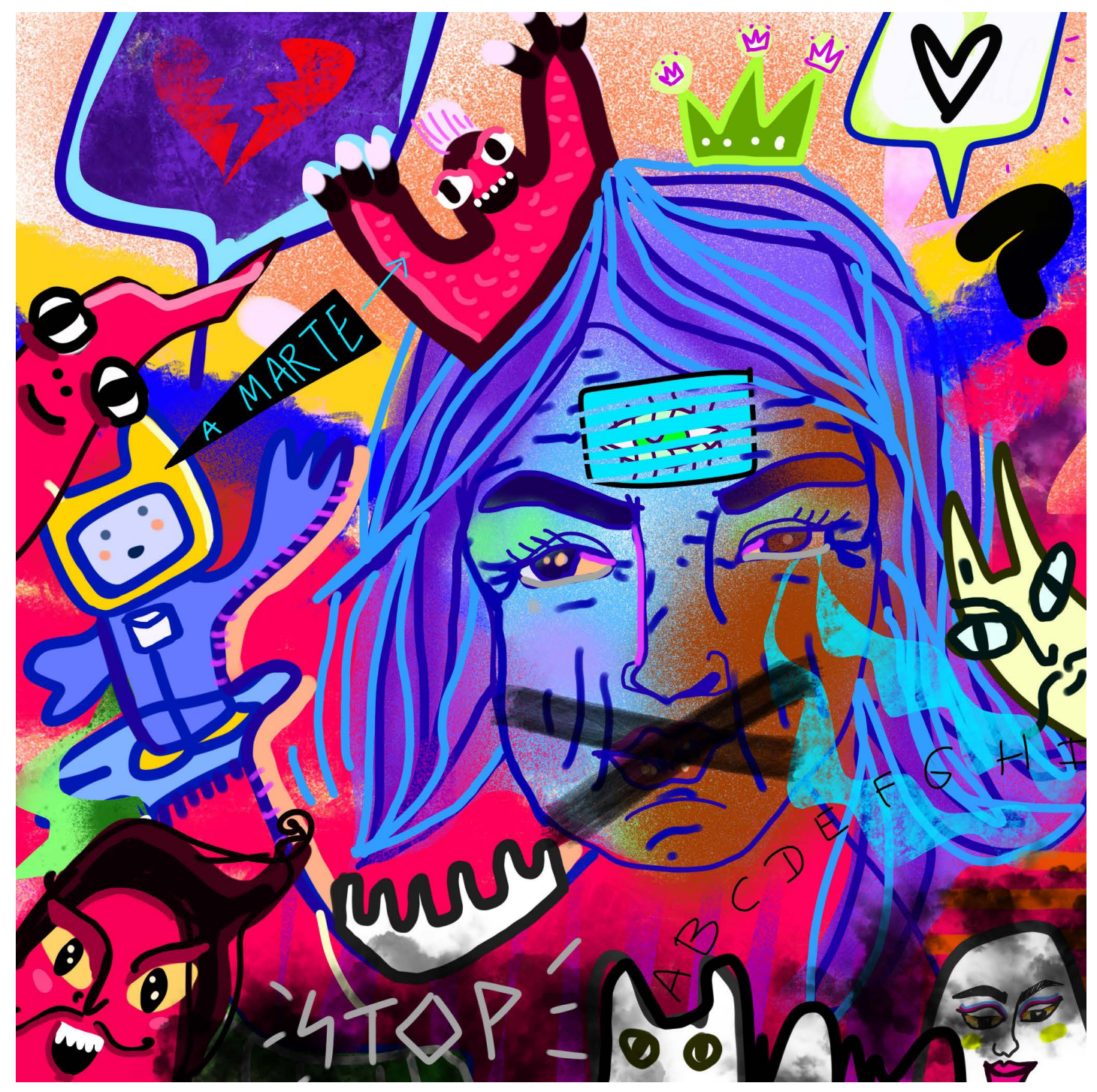

Artista invitado

Fabio Andrés Arboleda Mendoza

Stop

Ilustración digital

2020

Medellín 


\title{
Derechos sociales y género. Vinculación analítica de la migración femenina de retorno a México*
}

\author{
José Franco Aguilar (México)**
}

\section{Resumen}

Este artículo tiene como objetivo analizar las características de las mujeres retornadas a México mediante indicadores de derechos sociales y grupos específicos, contrastándolas con las de los retornados. Los indicadores resultan de los datos de la Encuesta Intercensal 2015 y se vinculan al contexto de operación de Somos Mexicanos, programa de apoyo a la reinserción de los migrantes de retorno. El análisis se desarrolla exponiendo los indicadores relacionados con los derechos sociales de los retornados. Sobre los indicadores de resultado se muestran contrastes entre la población retornada considerando el género, la etnia y a la población retornada afrodescendiente. Los resultados muestran la incidencia de estas categorías analíticas en las características particulares de las poblaciones analizadas. En este sentido, se concluye en la necesidad de que los programas de apoyo al retorno partan de este reconocimiento, dado que los retornados son un grupo heterogéneo con necesidades particulares específicas.

\section{Palabras clave}

Relaciones Internacionales; Políticas Públicas; Migración de Retorno; Derechos Sociales y Económicos; Género; Interseccionalidad.

Fecha de recepción: octubre de 2020

- $\quad$ Fecha de aprobación: abril de 2021

\section{Cómo citar este artículo}

Franco Aguilar, José. (2021). Derechos sociales y género. Vinculación analítica de la migración femenina de retorno a México. Estudios Políticos (Universidad de Antioquia), 62, pp. 52-78. https://doi.org/10.17533/udea.espo.n62a03

\footnotetext{
* Resultado del proceso de investigación del Programa de Becas Posdoctorales, marzo 2019-febrero 2020, Instituto de Investigaciones Jurídicas, Universidad Nacional Autónoma de México (UNAM).

${ }^{* *}$ Licenciado en Economía. Magíster en Población y Desarrollo. Doctor en Antropología. Investigador posdoctoral, Instituto de Investigaciones Jurídicas, Universidad Nacional Autónoma de México (UNAM). Correo electrónico: jfrancomx@gmail.com - Google Scholar: https://scholar.google.es/citat ions?user=tuFrpTkAAAAJ\&hl=es\&oi=ao
} 


\title{
Social Rights and Gender. Analytical Link of Migrant Women Returning to Mexico
}

\begin{abstract}
This article analyzes the characteristics of women who returned to Mexico through social rights indicators and specific groups, contrasting them with the characteristics of returnees. The indicators result from the data of the 2015 Intercensal Survey and are linked to the operating context of the "Somos Mexicanos" program to support the reintegration of return migrants. The analysis focuses on the indicators linked to the social rights of returnees. On the results indicators, contrasts are shown between the population returned considering gender, ethnicity, and the Afrodescendant population. The results show the incidence of these analytical categories in the particular characteristics of the populations analyzed. It is concluded that the return support program requires to acknowledge these particular characteristics of the populations, since the returnees are a heterogeneous group with specific needs.
\end{abstract}

\section{Keywords}

International Relations; Public Politics; Return Migration; Social and Economic Rights; Gender; Intersectionality. 


\section{Introducción}

El retorno, como característica de la movilidad humana, es un tema central en el panorama migratorio contemporáneo en México. Junto con la migración en tránsito, denotan la complejidad y diversidad del fenómeno migratorio en el país y se manifiestan como un reto significativo en términos de atención y acciones públicas encaminadas a atender dichas problemáticas. En cuanto al retorno se refiere, la reinserción e integración a la sociedad mexicana -individual y familiar - se muestran en la coyuntura actual como temas de análisis relevantes.

Bajo la política migratoria evidentemente antiinmigrante del del entonces presidente estadounidense Donald Trump, los mexicanos nacidos en territorio nacional y que actualmente viven en Estados Unidos se encontraron en una clara situación de vulnerabilidad. Para esta población, que, según las estimaciones de la Current Population Survey (CPS) alcanzan los 12.3 millones de personas mexicanas en 2018, y de las cuales la mitad se encuentran en una condición migratoria irregular, esto es, sin documentos migratorios legales (Conapo, 2017), el retorno - voluntario o forzado- se vislumbra como una posibilidad en su futuro. Así, el retorno asistido para quien

[ 54 ] regrese de forma voluntaria o forzada es fundamental, considerándose como una primera instancia que otorgue protección y apoyo en la consecución del pleno respeto a los derechos humanos, garantizando el desarrollo personal, la satisfacción de las necesidades básicas y la reinserción en la comunidad de forma óptima y segura de los mexicanos retornados.

En este contexto, en México se promovió la puesta en marcha de distintas acciones en favor de la población retornada, apoyos que han transitado desde una perspectiva meramente asistencial a una de facilitación del proceso de reinserción a partir de programas gubernamentales. En el ámbito nacional, se destacan: el Programa de Repatriación Humana (PRH) y el Procedimiento de Repatriación al Interior de la República (PRIM), implementados por el Instituto Nacional de Migración (INM). Sumado a los anteriores, la Organización Internacional de la Migración (OIM) ha impulsado el Programa de Atención a Migrantes Retornados (PAMIR) como un programa piloto que funcionó de manera conjunta con el PRIM.

Conjuntando los programas anteriores, el 30 de marzo del 2014 la Presidencia de la República anunció el lanzamiento del programa Somos 
Mexicanos dentro de la estrategia Somos mexicanos: Aquí tienes las puertas abiertas, la que contempló como objetivo:

[...] fortalecer la política del Estado mexicano orientada a la reinserción social de las personas mexicanas repatriadas al territorio nacional desde los Estados Unidos de América, así como sus vínculos sociales y culturales, en aras de que su regreso a México sea digno, productivo y benéfico, en provecho del bienestar familiar y del desarrollo regional y nacional (Secretaría de Gobernación, 6 de julio de 2016).

Esta estrategia se enmarca en una coyuntura en la que la política migratoria estadounidense era parte central del debate, y en un momento en el que la cantidad de mexicanos deportados era de 176968 personas (DHS, s. f.). La preocupación del Gobierno mexicano se reflejó en la concepción del programa mencionado, el cual ofrecía «apoyo integral a miles de connacionales que son repatriados de Estados Unidos, quienes llegan a México en condiciones de vulnerabilidad, luego de vivir por años en ese país dejando atrás a una familia y un patrimonio» (INM, 2016, diciembre 5). El programa se sustentaba bajo el respeto y pleno ejercicio de los principios fundamentales en materia de derechos humanos, y en este sentido dicha estrategia ha desarrollado un apoyo considerable a los retornados y repatriados. En 2016 el programa Somos Mexicanos apoyó a 200912 connacionales, facilitando su reinserción social y económica (INM, 2016, diciembre 5); sin embargo, es necesario considerar que, a pesar de los datos de los programas puestos en marcha por el Gobierno mexicano, la información disponible es insuficiente para hacer una valoración de las medidas tendientes al apoyo de retorno de mexicanos y de la gestión que se está haciendo de ellas. Sumado a lo anterior, no se dispone de información que permita conocer las implicaciones del programa en la reinserción de los retornados una vez que vuelven a su lugar de origen, ni detalles que posibiliten dar seguimiento a estos, así como al impacto de su retorno en las regiones y lugares de reasentamiento.

De esta manera, resulta primordial analizar los efectos de los programas asistenciales con el propósito de evidenciar sus limitaciones, y proponer nuevos lineamientos para el cumplimiento de los objetivos propuestos: una reinserción plena a las comunidades de origen. Asimismo, resulta relevante considerar las particularidades de la población retornada que, si bien desde las políticas públicas es considerada como una entidad única y homogénea, se distingue por ser una población heterogénea y, por tanto, demandante de una mirada analítica y conceptual no restrictiva que permita incorporar dicha diversidad. 
Así, en este trabajo el interés se enfoca en la situación de desventaja en la que se encuentran los migrantes de retorno en distintos ámbitos, lo que trae consigo que sean una población con diversas vulnerabilidades. ${ }^{1}$ Los retornados, principalmente los que regresan en condiciones forzadas -manifestándose de manera más drástica en los deportados-, son una población altamente vulnerable al ser su retorno involuntario y, por ello, proclive a que no exista la preparación y movilización de recursos. Esto explicaría, parcialmente, dicha condición de mayor vulnerabilidad, expresada en las dificultades que esta población ha experimentado en el proceso de reinserción social. La involuntariedad, como característica del retorno contemporáneo, se ha modificado: pasó de ser vista como una decisión tomada por los individuos a ser una opción política ejercida por los gobiernos a través de distintos mecanismos e intervenciones, que incluyen desde programas de fomento del retorno voluntario hasta políticas de deportaciones (Gandini, Lozano y Gaspar, 2015, p. 171). Así, esta mayor vulnerabilidad convierte a los migrantes de retorno en sujetos de política pública (CIDH, Caso Ximenes Lopes vs. Brasil, Sentencia 4 de julio de 2006, parágrafo 103).

Ahora bien, al considerar la vulnerabilidad del conjunto de los [ 56 ] retornados es evidente la necesidad de establecer programas asistenciales o políticas públicas universales que garanticen el cumplimiento de los derechos básicos de esta población, además de un conjunto de políticas o programas focalizados a los grupos más vulnerables, a fin de generar mayor equidad.

En este contexto, este artículo centra el análisis en las mujeres retornadas, considerando que son un colectivo con una doble vulnerabilidad, como migrantes de retorno y por su condición de género (Calavita, 2006). Así, las mujeres retornadas son un colectivo que, como regla general, está en condiciones de clara desigualdad con respecto al colectivo mayoritario. Por ello, se parte de que el género es la condición que determina que las mujeres estén en situación de especial vulnerabilidad en lo que a derechos humanos y asistencialismo gubernamental se refiere. Sin embargo, la vulnerabilidad

\footnotetext{
${ }^{1}$ Los grupos sociales en situación de vulnerabilidad, según la Ley General de Desarrollo Social de México (Artículo 5. ${ }^{\circ}$, fracción VI), son aquellos núcleos de población y personas que, por diferentes factores o la combinación de ellos, enfrentan situaciones de riesgo o discriminación que les impiden alcanzar mejores niveles de vida y, por lo tanto, requieren de la atención e inversión del Gobierno para lograr su bienestar.
} 
al interior del grupo también varía en función de otros factores como la raza y el color de la piel, la edad, la etnicidad, la clase socioeconómica, la cultura, la localización geográfica y el estatus como migrante, factores que se combinan para determinar la posición social de una persona y que destacan la preminencia de un análisis interseccional.

El objetivo del trabajo es analizar, mediante indicadores de derechos humanos y grupos específicos, las características de las mujeres retornadas a México, particularizando en indicadores de derechos sociales y contrastándolos con las características de los hombres retornados. Estos indicadores resultan de los datos de la Encuesta Intercensal 2015 (Inegi, 2015), ${ }^{2}$ y se encuentran en el contexto de operación del programa Somos Mexicanos.

Enfatizar y acotar el análisis a las mujeres retornadas constituye una manera de evidenciar cómo las ideologías, jerarquías y relaciones de género operan simultáneamente a través de diferentes ámbitos — micro, messo y macrosocial—, incrementando la vulnerabilidad de ellas — por su condición de retornadas y de género-. Dentro de estos ámbitos, la vulnerabilidad, entendida como aquella parte del riesgo más vinculada con las estructuras sociales que con las conductas individuales, se convierte en indicador clave para una aproximación y comprensión de las formas y dimensiones diferenciales de inequidad y desigualdad entre mujeres y hombres. En este sentido, las fronteras internacionales, espacios geográficos, socioeconómicos y culturales con identidad propia se constituyen en escenarios donde las vulnerabilidades acumulativas por razones de inequidad socioeconómica, étnica y de género se acentúan y agravan en contextos caracterizados, en muchas ocasiones, por la ausencia de recursos y la pérdida de los derechos más elementales (Mora, 2002). En consecuencia, la visibilidad de indicadores dentro del análisis del retorno femenino, derechos sociales y vulnerabilidad constituye una dimensión de análisis imprescindible, tanto para un abordaje integral de los derechos humanos como para visibilizar las consecuencias, en ocasiones ambiguas e incluso contradictorias, que las migraciones internacionales y los desplazamientos de personas pueden tener en las relaciones de género.

\footnotetext{
${ }^{2}$ La encuesta es referida al 15 de marzo de 2015. Tiene un tamaño de muestra esperado de 6.1 millones de viviendas y permite tener información en el ámbito nacional, entidad federativa, municipio y para cada una de las localidades con cincuenta mil o más habitantes.
} 
La importancia de la temática trasciende el ámbito mexicano, sin duda, el análisis de los derechos sociales evidencia la relevancia del reconocimiento normativo como fórmula para la atención especial de los grupos más vulnerables. Los derechos sociales en los Estados latinoamericanos han sido largamente desatendidos y violados, aún cuando las naciones se han comprometido a respetar, proteger y dar cumplimiento a estos, particularmente en los grupos más vulnerables.

\section{Derechos humanos y migración de retorno}

De acuerdo con el derecho internacional, ${ }^{3}$ se establece de manera inequívoca que los migrantes y sus familiares son, ante todo, seres humanos y titulares de derechos humanos universales, cuya dignidad y seguridad necesitan una protección especial. Así, como cualquier otra persona, todas las personas consideradas migrantes gozan de la protección del derecho internacional de los derechos humanos (OACDH, 2012).

Empero, hay situaciones en las que el tratamiento diferenciado es necesario, como en el caso de las mujeres, niños o miembros de minorías que necesitan protección especial porque están en una situación de mayor

[ 58 ] riesgo de discriminación o abuso. En este sentido, el objetivo principal al considerar el derecho internacional es lograr la igualdad de oportunidades y trato.

En la normativa internacional, la migración de retorno es un derecho. En el Artículo 8 inscrito en la parte ॥ de la Convención internacional sobre la protección de los derechos de todos los trabajadores migratorios y de sus familiares (ICRMW, por sus siglas en inglés) se establece: «Los trabajadores migratorios y sus familiares tendrán derecho a regresar en cualquier momento a su Estado de origen y permanecer en él» (Asamblea General de las Naciones Unidas. Resolución 45/148. 18 de diciembre de 1990). ${ }^{4}$ Asimismo, en los

\footnotetext{
${ }^{3}$ Los instrumentos internacionales de derechos humanos son esencialmente una serie de disposiciones jurídicas de mínimos elaboradas en conferencias internacionales para proporcionar orientación y, a menudo, un lenguaje específico a las legislaciones, políticas y prácticas nacionales. Los instrumentos internacionales comprenden tanto convenciones como declaraciones, aunque estas últimas no son vinculantes, a no ser que constituyan derecho internacional consuetudinario.

${ }^{4}$ Este derecho se reitera en disposiciones regionales de derechos humanos, como el Art. 2 del Protocolo número 4 al Convenio para la protección de los Derechos Humanos y de las Libertades Fundamentales de 1963 del Convenio europeo de derechos humanos de 1950; y en los artículos 12.1 y 2 de la Carta Africana de Derechos Humanos y de los Pueblos de 1981; asimismo, la importancia de este derecho se
} 
derechos sociales — derecho a un empleo y a un salario justo; a la protección social; a la vivienda; a la educación gratuita y de calidad; a la sanidad; a un entorno saludable; al acceso a la cultura; y a la alimentación- subyacen los elementos esenciales para una vida con dignidad y libertad. ${ }^{5}$

Los derechos sociales se establecen en el vinculante Pacto Internacional de Derechos Económicos, Sociales y Culturales (ICESCR, por sus siglas en inglés), ${ }^{6}$ en el cual el Comité de las Naciones Unidas de Derechos Económicos, Sociales y Culturales (CESCR, por sus siglas en inglés) reconoció explícitamente esta aplicabilidad «a todos los individuos, incluidos los no nacionales, como los refugiados, los solicitantes de asilo, los apátridas, los trabajadores migratorios y las víctimas de la trata internacional, independientemente de su condición jurídica y de la documentación que posean» (CESCR. E/C.12/GC/20. 2 de julio de 2009. Observación general N. ${ }^{\circ} 20$ ). Estos derechos son relevantes porque en ellos se establece un marco común de valores y normas universalmente reconocidos para movilizar los esfuerzos en favor del bienestar económico y social, la justicia, la participación política y la igualdad.

Alineado con lo anterior, los Objetivos de Desarrollo Sostenible (ODS) de la agenda de las Naciones Unidas para el desarrollo, la migración y los derechos vinculados a las personas migrantes son un tema manifiesto. La meta 8.8 incluida en el objetivo octavo, estipula la necesidad de «proteger los derechos laborales y promover un entorno de trabajo seguro y sin riesgos para todos los trabajadores, incluidos los trabajadores migrantes, en particular las mujeres migrantes y las personas con empleos precarios». Sumado a ello, el objetivo 10, «reducir la desigualdad en los países y entre ellos», se establece también en la meta 10.7: «facilitar la migración y la movilidad ordenadas, seguras, regulares y responsables de las personas,

ilustrada en el Caso de personas dominicanas y haitianas expulsadas Vs. República Dominicana. (28 de agosto de 2014). Serie C No. 282, párr. 389.

${ }^{5}$ Los derechos sociales surgen ante la necesidad de cumplir la ley en aquellos aspectos en los que las personas puedan sentirse desprotegidas o en los casos en que carezcan de reconocimiento dentro de su comunidad, población, nación, país, grupo o sociedad. El derecho social es netamente reivindicativo, en especial con aquellos colectivos que han sido excluidos por alguna circunstancia social o política: indígenas, niños, afrodescendientes, mujeres, personas con alguna discapacidad y otros grupos minoritarios (ACNUR Comité Español, 2016, junio).

${ }^{6}$ A fecha de 12 de agosto de 2015, 164 países habían ratificado el ICESCR - México firmó y ratificó dicho pacto-, aceptando la obligación de respetar, proteger y satisfacer los derechos económicos, sociales y culturales de todas las personas en sus territorios (Asamblea General de las Naciones Unidas. Resolución 2200 A (XXI). 3 de enero de 1976). 
incluso mediante la aplicación de políticas migratorias planificadas y bien gestionadas» (Undesa, s. f.).

En México, la Constitución Política en su Art. 1 expresa que todas las personas en los Estados Unidos Mexicanos - que ingresan, transitan, salen y retornan a territorio nacional- gozan de los derechos humanos reconocidos en esta y en los tratados internacionales de los que el Estado mexicano es parte. Sobre el retorno, en la Ley General de Población se establece en el capítulo $v$ elementos referentes a los emigrantes nacionales que regresan al país. En el Art. 3 se alude a los derechos sociales al volver: «las acciones de atención y reintegración de mexicanos repatriados, poniendo especial énfasis en que sean orientados acerca de las opciones de empleo y vivienda que haya en el lugar del territorio nacional en el que manifiesten su intención de residir».

En este mismo tenor se pronuncia la Ley de Migración del 25 de mayo de 2011. En el Art. 2 se menciona como un principio en el que se sustenta la política migratoria mexicana: «[...] facilitar el retorno al territorio nacional y la reinserción social de los emigrantes mexicanos y sus familias, a través de programas interinstitucionales y de reforzar los vínculos entre las

[ 60 ] comunidades de origen y destino de la emigración mexicana, en provecho del bienestar familiar y del desarrollo regional y nacional». Lo anterior parte del Plan Nacional de Desarrollo 2013-2018 y del Plan Especial de Migración 2014-2018, establecidos por la administración de Enrique Peña Nieto. En ellos se enfatizaba promover los procesos de integración y reintegración de las personas migrantes y sus familias, creando mecanismos y fortaleciendo los programas de reinserción. ${ }^{7}$

En este contexto, la protección a los retornados y la importancia del ejercicio de sus derechos son parte fundamental del entramado jurídico nacional e internacional, así como parte de un enfoque central en el que se basan los ODS. La estrategia nacional Somos mexicanos, apegada a los principios fundamentales en materia de derechos humanos, se circunscribió a esta retórica nacional e internacional con el objetivo de lograr «la integración y el bienestar familiar de las y los migrantes mexicanos repatriados. Lo anterior, para el desarrollo regional y nacional».

\footnotetext{
${ }^{7}$ En el actual Plan Nacional de Desarrollo 2019-2024, en el apartado Migración: soluciones de raíz, no se menciona acciones para atender a los retornados, no se estipula el tema.
} 
En este panorama y en la coyuntura en la que operaba el programa -2014-2019_, se analizan diversos indicadores de derechos humanos en mujeres retornadas con distintas características, en concreto: el acceso a la salud, la educación, la vivienda y el trabajo.

\section{Metodología}

La concepción metodológica del artículo se sustenta en los indicadores de derechos humanos establecidos por la Oficina del Alto Comisionado de las Naciones Unidas para los Derechos Humanos (OACDH, 2012), que se precisan como:

[...] informaciones concretas sobre el estado o la condición de un objeto, un acontecimiento, una actividad o un resultado que pueden estar relacionados con las normas de derechos humanos; que abordan y reflejan principios e intereses en materia de derechos humanos y que se utilizan para evaluar y vigilar la promoción y protección de los derechos humanos (p. 19).

De esta forma, se asignan características o atributos específicos a cada derecho humano que exhiba su pleno significado. Los atributos de los derechos humanos identificados por la OACDH pueden ser adaptados para grupos específicos, entre ellos, los migrantes. La metodología ofrecida por esta especifica que hay tres tipos de indicadores: estructurales, de procesos y de resultados. Estos miden los compromisos de los Estados de cumplir sus obligaciones - jurídicas y de políticas - en materia de derechos humanos, los resultados de tales compromisos y aspectos de los procesos en los que se fundamentan. Las características de cada indicador son:

- Los indicadores estructurales se centran en el marco jurídico y de políticas establecido. Entre estos se encuentran los tratados internacionales ratificados y el compromiso normativo vigente del Estado.

- Los indicadores de proceso intentan captar las medidas de política del Estado con hitos que puedan consolidar y dar lugar a los resultados de derechos humanos deseados. Se consideran aspectos tales como política pública específica, programas de gobierno y recursos establecidos para hacer efectivo el derecho.

- Los indicadores de resultados miden los resultados reales, logros individuales y colectivos que reflejan el estado de disfrute de los derechos humanos en determinado contexto. 
La metodología desarrollada por la $\mathrm{OACDH}$ ofrece un enfoque estructurado que traduce normas universales de derechos humanos en indicadores que, en el ámbito nacional, son contextualmente pertinentes.

Al considerar los indicadores expuestos, el análisis se desarrolla exponiendo los tres tipos de indicadores vinculados a los derechos sociales enmarcados en el contexto de la estrategia Somos Mexicanos referida a la población retornada, mostrando contrastes entre hombres y mujeres, y según características particulares de la población —resto de los retornados, población afrodescendiente e indígena-.

La fuente de datos para los indicadores de resultados es la Encuesta Intercensal 2015 (Inegi, 2015), la cual ofrece información sobre las personas que vivían en Estados Unidos cinco años atrás. Se consideran como migrantes de retorno a quien declaró tener nacionalidad mexicana, residir cinco años antes en Estados Unidos y al momento de la encuesta radicar en territorio nacional. La Encuesta se utiliza porque ofrece información de temas referentes a los derechos socioeconómicos —educación, empleo, salud y vivienda-.

[ 62 ] Es importante destacar que la Encuesta Intercensal 2015 no ofrece información que vincule directamente a los sujetos de estudio — retornadoscon la estrategia Somos Mexicanos, por lo que se considera una vinculación indirecta y contextual. La Encuesta está referida al 15 de marzo de 2015 y la estrategia se inició el 26 de marzo de 2014. En este sentido, la población retornada captada por la fuente de información se encuentra en una coyuntura donde opera el programa gubernamental. Considerando la posibilidad de que los retornados no vuelvan a emigrar fuera del país, la encuesta tiene una alta probabilidad de mostrar las características del padrón de beneficiados de la estrategia -200 912 en 2016, según cifras del INM (2016, diciembre 5)—. Así, las características sociodemográficas de los y las retornadas que obtuvieron algún apoyo de la estrategia probablemente se ven reflejadas en los datos, considerando que la encuesta es el proyecto estadístico con datos más actualizados de información sociodemográfica del país en el periodo intercensal comprendido entre 2010 y $2020 .^{8}$

\footnotetext{
${ }^{8}$ La Encuesta Intercensal 2015 (Inegi, 2015) es un proyecto de cobertura temática amplia, cuyo diseño permite proporcionar estimaciones en el ámbito nacional, por entidad federativa, municipio y para todas las localidades de cincuenta mil o más habitantes.
} 
De esta manera, este acercamiento metodológico intenta evidenciar, a través del desglose tanto de las mujeres como de los hombres en la migración de retorno a México, las situaciones de exclusión y desigualdad respecto a sus derechos sociales, expresados a través de indicadores de resultado disponibles con la información de la fuente de datos analizada.

\section{Migración de retorno, género e indicadores de derechos humanos}

La importancia de la reinserción social y económica de los retornados vinculada con el desarrollo local se ha puesto de manifiesto en la presente década en México (Gandini, Lozano y Gaspar, 2015; García y Del Valle, 2016; El Colegio de México y Comisión Nacional de Derechos Humanos, 2019). En este contexto, evidenciar la disparidad en el ejercicio de los derechos sociales de la población retornada en relación con el género fomenta la utilización de objetivos e indicadores mensurables para supervisar la protección de los y las migrantes, para una aproximación y comprensión de las formas y dimensiones diferenciales de inequidad y desigualdad entre mujeres y hombres. La información visibiliza las características que deben atenderse en términos de derechos humanos para mejorar los programas públicos, buscando incidir en las condiciones individuales de los y las retornadas, pero también establece la pauta para que la sociedad se beneficie de los recursos —económicos, de capital humano, culturales e identitarios- que poseen los retornados.

Los indicadores referidos a la migración de retorno en el contexto particular de la estrategia Somos Mexicanos se muestran en el cuadro 1. Los indicadores muestran desde un ámbito general -indicadores estructurales- a uno particular —indicadores de resultado-, las medidas adoptadas por los Estados para cumplir sus obligaciones, ya sea respetar, proteger o hacer efectivos los derechos sociales de la población retornada. Los indicadores estructurales reflejan la ratificación, la adopción de instrumentos legales y la existencia de mecanismos institucionales básicos que se consideran necesarios para la promoción y la protección de los derechos humanos de la población analizada. Se indican normas internacionales y el marco jurídico en el que se basa la estrategia que protege sus derechos sociales. 
Cuadro 1. Indicadores sobre los derechos socioeconómicos de la población retornada en México.

\begin{tabular}{|c|c|}
\hline \begin{tabular}{|l|} 
Indicadores \\
estructurales
\end{tabular} & $\begin{array}{l}\text { - Del derecho internacional: } \\
\text { - Convención internacional sobre la protección de los derechos de todos los } \\
\text { trabajadores migratorios y de sus familiares. } \\
\text { - Pacto Internacional de Derechos Económicos, Sociales y Culturales. } \\
\text { - Objetivos de Desarrollo Sostenible (ODS). } \\
\text { - Del ámbito nacional: } \\
\text { - Constitución Política de los Estados Unidos Mexicanos. } \\
\text { - Ley General de Población (art. 83). } \\
\text { - Ley Orgánica de la Administración Pública Federal (art. 27, fracción XLI). } \\
\text { - Ley de Migración (art. 2). }\end{array}$ \\
\hline $\begin{array}{l}\text { Indicadores } \\
\text { de proceso }\end{array}$ & $\begin{array}{l}\text { - Estrategia Somos mexicanos: Aquí Tienes las Puertas Abiertas, que tiene como } \\
\text { misión facilitar la reinserción social y económica de las personas repatriadas } \\
\text { mexicanas con el fin de que su retorno al país sea digno, productivo y apegado } \\
\text { a los principios fundamentales en materia de derechos humanos (INM, s. f. a). } \\
\text { El programa pretende incidir y tiene como categorías de atención: } \\
\text { - Acceso a servicios básicos de alimentación y salud. } \\
\text { - Trámites de documentos de identidad. } \\
\text { - Incidencia en el ámbito laboral: Bolsa de trabajo, programas de autoempleo. } \\
\text { - Acceso a opciones para continuar estudios (INM, s. f. b). }\end{array}$ \\
\hline $\begin{array}{l}\text { Indicadores } \\
\text { de resultado }\end{array}$ & $\begin{array}{l}\text { - Relativo al derecho a la identidad } \\
\text { - Porcentaje de población retornada que tiene acta de nacimiento o está } \\
\text { inscrita(o) en el registro civil mexicano. } \\
\text { - Relativos al derecho a la vivienda } \\
\text { - Porcentaje de población retornada según tenencia de vivienda. } \\
\text { - Disponibilidad de servicios. } \\
\text { - Promedio de ocupantes en la vivienda. } \\
\text { - Relativos al derecho a la educación } \\
\text { - Tasas de analfabetismo entre jóvenes (15-24 años) y adultos ( } 25 \text { y más) } \\
\text { - retornados. } \\
\text { - Población retornada de } 15 \text { y más años según nivel de escolaridad. } \\
\text { - Pelativos al derecho a la salud } \\
\text { - Porcentaje de población retornada según afiliación a servicios de salud. } \\
\text { - Relativos al derecho al trabajo } \\
\text { - Porcentaje de la población retornada según condición de actividad } \\
\text { - económica. } \\
\text { - Ingresos mensualizados promedio. } \\
\text { - Características de la población inactiva. } \\
\text { - Características de la situación laboral de la población ocupada. } \\
\text { - Proporción de la población económicamente activa según prestaciones } \\
\text { laborales. } \\
\text { - Proporción de retornados según trabajo no remunerado. }\end{array}$ \\
\hline
\end{tabular}

Fuente: elaboración propia a partir de (Inegi, 2015). 
Los indicadores de proceso se conjugan en la estrategia Somos Mexicanos, la cual concentra el esfuerzo realizado por el Estado para transformar sus compromisos en materia de derechos sociales para los retornados en los resultados deseados. La estrategia como medida de política pública se muestra en función de una relación causa-efecto implícita y como paso intermedio entre el compromiso adquirido por el Estado y los resultados mostrados.

Los indicadores de resultado miden los efectos del programa en determinado momento. En el siguiente apartado se profundiza en los atributos de los derechos sociales y se identifican indicadores que puedan relacionarse estrechamente con el disfrute de ese derecho o atributo. Los resultados se muestran en función de la información obtenida de la fuente de datos y se desagregan según sexo y características diferenciadas asociadas a la vulnerabilidad.

\section{Indicadores de derechos sociales para los retornados}

En México, según la Encuesta Intercensal 2015 (Inegi, 2015), había 487 594 migrantes de retorno procedentes de Estados Unidos, principal país del que regresa el grueso de la población mexicana. ${ }^{9}$ La entidad federativa con mayor cantidad de retornados es Jalisco con 9,2\%, le siguen Michoacán con $7,8 \%$, Baja California con $7,1 \%$ y Guanajuato con $6,5 \%$. Respecto al volumen por sexo, 64,6\% fueron hombres (243 653) y 35,4\% mujeres (133 483).

En consonancia con la idea de derechos sociales y vulnerabilidad de género, también se suma, desde una perspectiva interseccional, la consideración de la población indígena y afrodescendiente, poblaciones que históricamente muestran condiciones de vida asociadas a mayor vulnerabilidad. Su incorporación al análisis permitirá visibilizar problemáticas que les afectan particularmente. Aunado a la visión de género y la incipiente apertura al análisis de la población afrodescendiente en México, estas categorías analíticas pretenden constituir en actores protagónicos a las poblaciones que acumulan vulnerabilidades con la temática del retorno.

En la tabla 1 se muestran datos generales de las poblaciones de retornados analizadas. ${ }^{10}$ Puede observarse que la distribución obedece a un patrón masculino: la relación hombres-mujeres que manifiesta la cantidad

\footnotetext{
${ }^{9}$ El segundo país del que regresa la población mexicana, según información de la fuente de datos consultada, es Canadá, con un volumen poblacional de 7985 retornados.

${ }^{10} \mathrm{Con}$ el fin de analizar las características de poblaciones excluyentes, para exhibir de mejor forma las diferencias, se establecieron las siguientes delimitaciones: población retornada no afro ni indígena (377
} 
de hombres por cada cien mujeres. Entre la población indígena esta relación es de 267 hombres por cada 100 mujeres, mientras que en el resto de los retornados - no indígenas, ni afrodescendientes- 182 por cada 100. La edad mediana de los distintos grupos oscila entre los 32 y 36 años, siendo los hombres indígenas el grupo de mayor edad.

Tabla 1. Población migrante de retorno, datos generales.

\begin{tabular}{|l|c|c|c|c|c|c|}
\hline $\begin{array}{c}\text { Datos demográficos } \\
\text { generales }\end{array}$ & Hombres & Mujeres & $\begin{array}{c}\text { Hombres } \\
\text { afro }\end{array}$ & $\begin{array}{c}\text { Mujeres } \\
\text { afro }\end{array}$ & $\begin{array}{c}\text { Hombres } \\
\text { indígenas }\end{array}$ & $\begin{array}{c}\text { Mujeres } \\
\text { indígenas }\end{array}$ \\
\hline Absoluto & 243653 & 133483 & 5807 & 2755 & 74162 & 27734 \\
\hline$\%$ & 64,6 & 35,4 & 67,8 & 32,2 & 72,8 & 27,2 \\
\hline $\begin{array}{l}\text { Relación hombres y } \\
\text { mujeres }\end{array}$ & \multicolumn{2}{|c|}{182.5} & \multicolumn{2}{|c|}{210.8} & \multicolumn{2}{c|}{267.4} \\
\hline Edad mediana & 34.8 & 32.4 & 33.1 & 32.1 & 35.9 & 33.6 \\
\hline $\begin{array}{l}\text { Razón de dependencia } \\
\text { económica }\end{array}$ & \multicolumn{2}{|c|}{18.9} & \multicolumn{2}{|c|}{19.7} & \multicolumn{2}{|c|}{12.2} \\
\hline
\end{tabular}

Fuente: elaboración propia a partir de (Inegi, 2015).

Respecto a la dependencia económica, ${ }^{11}$ para la población retornada afrodescendiente existen 20 personas en edad de dependencia por cada 100 en edad productiva. La razón es de casi 1 y 8 puntos por encima de lo observado para el total general y para el total de indígenas, respectivamente.

Los indicadores de resultado relativos al derecho a la identidad, a la vivienda y a la educación se muestran en la tabla 2 . Al considerar el indicador tenencia de acta de nacimiento, relativo al derecho a la identidad, se destaca que la mayoría posee el documento; sin embargo, es relevante la población que se declara como registrada en otro país, lo cual abre la posibilidad de personas con doble nacionalidad, pero sin un acta de nacimiento nacional. En el caso de las mujeres afrodescendientes retornadas, esta condición corresponde a $2,3 \%$. La población retornada indígena es la que manifiesta la mayor posesión del documento.

133), población afrodescendiente (8562) y población que se considera indígena no afrodescendiente (101 896).

${ }^{11}$ Es un indicador teórico de dependencia económica potencial, mide la población en edades inactivas - menores de 15 años y mayores de 65-con relación a la población en edades activas -15 a 64 años-. 
Derechos sociales y género. Vinculación analítica de la migración femenina...

Tabla 2. Población migrante de retorno, indicadores de resultados.

\begin{tabular}{|c|c|c|c|c|c|c|}
\hline & Hombres & Mujeres & $\begin{array}{l}\text { Hombres } \\
\text { afro }\end{array}$ & $\begin{array}{c}\text { Mujeres } \\
\text { afro }\end{array}$ & $\begin{array}{l}\text { Hombres } \\
\text { indígenas }\end{array}$ & $\begin{array}{l}\text { Mujeres } \\
\text { indígenas }\end{array}$ \\
\hline \multicolumn{7}{|c|}{ Acta de nacimiento o registro en el país (vinculado al derecho a la identidad) \% } \\
\hline Tiene acta & 97,7 & 97,3 & 97,5 & 96,5 & 98,8 & 98,3 \\
\hline No tiene & 0,7 & 0,6 & 0,4 & 0,3 & 0,5 & 0,4 \\
\hline $\begin{array}{l}\text { Esta registrado en otro } \\
\text { país }\end{array}$ & 1,3 & 1,8 & 1,5 & 2,3 & 0,4 & 1,1 \\
\hline No especificado & 0,3 & 0,3 & 0,6 & 0,9 & 0,3 & 0,2 \\
\hline \multicolumn{7}{|c|}{ Relativos al derecho a la vivienda } \\
\hline \multicolumn{7}{|c|}{ Tenencia de vivienda \% } \\
\hline Propia & 60,6 & 60,3 & 66,5 & 65,9 & 69,8 & 68,4 \\
\hline Rentada & 21,6 & 22,4 & 17,2 & 16,9 & 15 & 15,9 \\
\hline $\begin{array}{l}\text { De un familiar o } \\
\text { prestada }\end{array}$ & $15 ., 9$ & 15,4 & 15,4 & 16,7 & 13,7 & 13,7 \\
\hline Otra situación & 1,5 & 1,3 & 0,5 & 0,2 & 1,2 & 1,6 \\
\hline N/E & 0,4 & 0,6 & 0,3 & 0,3 & 0,3 & 0,4 \\
\hline \multicolumn{7}{|c|}{ Disponibilidad de servicios \% } \\
\hline Agua entubada & 85,7 & 89,2 & 79,6 & 86,9 & 80,5 & 83,6 \\
\hline Drenaje & 75,4 & 80,6 & 53,7 & 63,7 & 58,6 & 66,4 \\
\hline Servicio sanitario & 94,7 & 96,8 & 88,3 & 93,3 & 86,9 & 91,4 \\
\hline Promedio de personas & 2 & 2,5 & 1,9 & 2,4 & 1,8 & 2,4 \\
\hline \multicolumn{7}{|c|}{ Relativos al derecho a la educación } \\
\hline \multicolumn{7}{|c|}{ Población de 15 y más, según nivel de escolaridad \% } \\
\hline Sin escolaridad & 2,4 & 2,7 & 3,6 & 2,4 & 3,4 & 3,7 \\
\hline Básica & 64,6 & 55,2 & 69 & 64,3 & 73,4 & 65,1 \\
\hline Media superior & 23,4 & 26,6 & 19,1 & 21,3 & 18,2 & 22,7 \\
\hline Superior & 9,4 & 15,3 & 8,1 & 11,9 & 4,9 & 8,4 \\
\hline No especificado & 0,2 & 0,2 & 0,2 & 0,1 & 0,1 & 0,1 \\
\hline \multicolumn{7}{|c|}{ Analfabetismo \% } \\
\hline $\begin{array}{l}\text { Analfabetismo de } 15 \\
\text { años y más }\end{array}$ & 2,6 & 0,7 & 4,4 & 2,9 & 0,7 & 5,8 \\
\hline
\end{tabular}

Fuente: elaboración propia a partir de (Inegi, 2015). 
Sobre el derecho a la vivienda se muestran como indicadores de resultado la tenencia de la vivienda y la disponibilidad de servicios. Con relación a la tenencia, destaca que entre 60 y 70\% de la población retornada habita en una vivienda propia, siendo las mujeres del resto de los retornados el grupo que menor proporción obtuvo en este rubro $(60,3 \%)$, y el que más habita en una vivienda rentada $(22,4 \%)$.

La disponibilidad de servicios en la vivienda se relaciona directamente con la salud y bienestar de sus residentes, por ello la importancia de su análisis. Al considerar la disponibilidad de agua entubada y drenaje, en las viviendas afrodescendientes se observa una disminución en el valor de cada uno de ellos, es decir, son las poblaciones con menor acceso a estos servicios, tanto hombres como mujeres. El drenaje es el servicio en el que más diferencia obtuvieron al registrar un valor casi $20 \%$ menor respecto al resto de los retornados. En general, fueron las viviendas de los hombres retornados afrodescendientes las que presentaron un menor acceso a la disposición de servicios. El promedio de personas en las viviendas de mujeres retornadas es mayor respecto a los hombres, sin distinción de las poblaciones examinadas.

[ 68 ] Por otra parte, los indicadores relativos al derecho a la educación, reconocidos como fundamentales por la fuerte influencia en muchos otros ámbitos de la vida de las personas, muestran datos relevantes. Los datos sobre el nivel de escolaridad para la población de 15 años y más muestran que el grueso de la población retornada posee educación básica, aunque son los hombres en general quienes obtuvieron un mayor porcentaje en el nivel de escolaridad. Es destacable que, a mayor nivel educativo — niveles medio superior y superior-, las mujeres retornadas tienen superioridad porcentual respecto a los varones, lo cual representa que están más escolarizadas.

El analfabetismo es una condición que contribuye a la desigualdad social. De acuerdo con las cifras, el analfabetismo se manifiesta con mayor intensidad entre la población de mujeres indígenas retornadas, porcentualmente la población con un mayor grado de esta condición. Las diferencias entre sexos son marcadas entre poblaciones, evidenciando un acceso a la escolaridad marcado por el género y, seguramente, asociado a la menor cobertura de los servicios educativos en el pasado. 
En la tabla 3 se presentan indicadores respecto al derecho a la salud y al trabajo. Respecto a la afiliación a servicios de salud, la mayoría de la población retornada se encuentra afiliada a instituciones públicas de salud. ${ }^{12}$ El porcentaje más alto de afiliación lo muestran las mujeres afrodescendientes $(69,5 \%)$ y las indígenas $(68,7 \%)$, posiblemente, debido a la ampliación del Seguro Popular en zonas marginadas. La población con mayor proporción de no afiliación son los hombres en general, independientemente de su adscripción; mientras que los hombres afrodescendientes alcanzan los valores más elevados $(36,9 \%)$ de no afiliación a servicios de salud.

Tabla 3. Población migrante de retorno, indicadores de resultados.

\begin{tabular}{|c|c|c|c|c|c|c|}
\hline & Hombres & Mujeres & $\begin{array}{c}\text { Hombres } \\
\text { Afro }\end{array}$ & $\begin{array}{c}\text { Mujeres } \\
\text { Afro }\end{array}$ & $\begin{array}{l}\text { Hombres } \\
\text { indígenas }\end{array}$ & $\begin{array}{l}\text { Mujeres } \\
\text { indígenas }\end{array}$ \\
\hline \multicolumn{7}{|c|}{ Relativos al derecho a la salud } \\
\hline \multicolumn{7}{|c|}{ Afiliación a servicios de salud \% } \\
\hline $\begin{array}{l}\text { Afiliada a instituciones } \\
\text { públicas }\end{array}$ & 60,1 & 66,6 & 60 & 69,5 & 63,1 & 68,7 \\
\hline $\begin{array}{l}\text { Afiliada a instituciones } \\
\text { privadas }\end{array}$ & 2,8 & 3,9 & 1,4 & 3,1 & 0,8 & 1,8 \\
\hline Otra institución & 1,3 & 1,6 & 1,5 & 1,3 & 0,6 & 1,3 \\
\hline No afiliado(a) & 35,5 & 27,6 & 36,9 & 26,1 & 35,3 & 27,9 \\
\hline No especificado & 0,3 & 0,3 & 0,2 & 0 & 0,2 & 0,3 \\
\hline \multicolumn{7}{|c|}{ Carencia alimentaria \% } \\
\hline $\begin{array}{l}\text { Falta de alguna comida } \\
\text { al día (adultos) por falta } \\
\text { de dinero }\end{array}$ & 8,2 & 7,1 & 14,4 & 10,2 & 10,9 & 11,1 \\
\hline \multicolumn{7}{|c|}{ Relativos al derecho al trabajo } \\
\hline \multicolumn{7}{|c|}{ Características económico-laborales \% } \\
\hline $\begin{array}{l}\text { Población } \\
\text { económicamente activa } \\
\text { (PEA) }\end{array}$ & 76,9 & 32,8 & 82,3 & 38,7 & 79,6 & 31,8 \\
\hline $\begin{array}{l}\text { Población } \\
\text { económicamente } \\
\text { inactiva (PEI) }\end{array}$ & 22,9 & 67,1 & 17,5 & 60,9 & 20,3 & 68,1 \\
\hline No especificado & 0,2 & 0,1 & 0,2 & 0,4 & 0,1 & 0,1 \\
\hline
\end{tabular}

${ }^{12}$ En esta categoría se incluye al Seguro Popular o para una Nueva Generación (Siglo XxI), el Instituto Mexicano del Seguro Social (IMSS), el Seguridad y Servicios Sociales de los Trabajadores del Estado (ISSSTE), el ISSSTE estatal y Petróleos Mexicanos (Pemex), Defensa o Marina. 
Tabla 3. (Continuación).

\begin{tabular}{|l|c|c|c|c|c|c|c|}
\hline & Hombres & Mujeres & $\begin{array}{c}\text { Hombres } \\
\text { Afro }\end{array}$ & $\begin{array}{c}\text { Mujeres } \\
\text { Afro }\end{array}$ & $\begin{array}{c}\text { Hombres } \\
\text { indígenas }\end{array}$ & $\begin{array}{c}\text { Mujeres } \\
\text { indígenas }\end{array}$ \\
\hline \multicolumn{7}{|c|}{ Características de la PEI \% } \\
\hline Estudiante & 26,8 & 17,5 & 20,7 & 16,7 & 14,5 & 13,5 \\
\hline Pensionados o jubilados & 8,8 & 1,9 & 2,7 & 1,8 & 5,5 & 1,6 \\
\hline Quehaceres del hogar & 4,0 & 72,9 & 3,8 & 67,2 & 5,1 & 78,2 \\
\hline $\begin{array}{l}\text { Con alguna limitación } \\
\text { física o mental }\end{array}$ & 5,4 & 1,3 & 6,0 & 1,5 & 5,4 & 1,1 \\
\hline $\begin{array}{l}\text { Otras actividades no } \\
\text { económicas }\end{array}$ & 55,0 & 6,4 & 66,8 & 12,8 & 69,4 & 5,6 \\
\hline \multicolumn{7}{|c|}{ Características de la población ocupada \% } \\
\hline $\begin{array}{l}\text { Trabajadores } \\
\text { asalariados }\end{array}$ & 70,4 & 69,5 & 63,5 & 63,2 & 63,7 & 63,0 \\
\hline Empleadores & 4,7 & 4,3 & 4,0 & 5,9 & 3,5 & 3,3 \\
\hline $\begin{array}{l}\text { Trabajadores por cuenta } \\
\text { propia }\end{array}$ & 20,9 & 22,9 & 24,6 & 26,3 & 24,8 & 28,8 \\
\hline Trabajadores sin pago & 3,5 & 1,9 & 7,3 & 3,8 & 7,4 & 3,2 \\
\hline No especificado & 0,5 & 1,4 & 0,6 & 0,8 & 0,6 & 1,7 \\
\hline \multicolumn{7}{|c|}{ Ingresos promedio mensualizados \% } \\
\hline Hasta 1 SM & 3,8 & 4,3 & 5,1 & 3,1 & 6,4 & 9,3 \\
\hline Más de 1 a 3 SM & 34,2 & 24,7 & 36,7 & 32,9 & 40,6 & 30,6 \\
\hline Más de 3 SM & 53,0 & 64,6 & 45,4 & 46,4 & 40,6 & 55,0 \\
\hline
\end{tabular}

Fuente: elaboración propia a partir de (Inegi, 2015).

Vinculado a cuestiones de salud, se incluye el estimado del porcentaje de personas adultas que residen en la vivienda y que por falta de dinero no tuvieron alguna comida al día. ${ }^{13}$ Los porcentajes mayoritarios de población que sufrió esta carencia fueron los hombres afrodescendientes $(14,4 \%)$ y las mujeres indígenas $(11,1 \%)$.

En la tabla 3 también se presentan los primeros indicadores relacionados con la actividad laboral. El primer indicador es la división entre población

\footnotetext{
${ }^{13}$ La temática que quiere captar la Encuesta Intercensal 2015 es sobre la alimentación. La pregunta específica sobre la que se desarrolla este indicador es: «De diciembre a la fecha, ¿alguna vez, algún adulto que vive en esta vivienda dejó de desayunar, comer o cenar por falta de dinero?» (Inegi, 2015).
} 
económicamente activa (PEA) e inactiva (PEI). ${ }^{14}$ La PEA está directamente vinculada al género: los hombres en su mayoría exhiben mayores niveles de inserción al mercado de trabajo o deseos de insertarse. La PEA de los retornados afrodescendientes alcanza $82,3 \%$, la más alta, seguida por los retornados indígenas con $79,6 \%$. Por su parte, las mujeres tienen mayor porcentaje en la PEI. Las mujeres indígenas tienen el mayor porcentaje $(68,1 \%)$, seguidas por las mujeres del resto de retornados $(67,1 \%)$. Al precisar las actividades que desarrollan las personas que hacen parte de la $\mathrm{PEI}$, para las mujeres los mayores porcentajes se ubican en los «quehaceres del hogar». Casi 8 de cada 10 mujeres indígenas mencionan esta actividad $(78,2 \%)$. Los estudiantes son representados mayoritariamente por hombres, los varones del resto de los retornados son los que tienen mayor presencia porcentual en este rubro $(26,8 \%)$, aunque no son tan drásticas las diferencias. El grupo menos representado en la categoría estudiante son las mujeres indígenas con solo 13,5\%. En los varones resalta que las denominadas otras actividades no económicas que, en síntesis, se refieren simplemente a no trabajar, obtienen la mayor presencia porcentual, alcanzando 69,4\% del total de hombres indígenas.

Por otro lado, las personas que hacen parte de la población ocupada - trabajadores al momento de la encuesta- según posición en el trabajo, presentan como característica generalizada laborar como trabajadores asalariados, sin distinción de poblaciones o sexo; no obstante, son los hombres del resto de retornados los que alcanzan el mayor porcentaje $(70,4 \%)$. La segunda categoría con mayor presencia porcentual de las poblaciones analizadas es trabajadores por cuenta propia, en esta las mujeres retornadas indígenas y afrodescendientes poseen el mayor porcentaje (28,8\% y $26,3 \%$, respectivamente). Las mujeres afrodescendientes que son empleadoras obtuvieron 5,9\%, la mayor representación porcentual de esta dimensión. Las personas que trabajan pero que no obtienen remuneración -trabajadores sin pago-, son proporcionalmente mayor en la comunidad indígena y afrodescendiente: los hombres indígenas alcanzan $7,4 \%$ y los afrodescendientes $7,3 \%$.

\footnotetext{
${ }^{14}$ La PEA la integran todas las personas de 12 y más años que realizaron algún tipo de actividad económica — población ocupada— o que buscaron activamente hacerlo — población desocupada abierta - en los dos meses previos a la semana de levantamiento. La PEI, como contraparte, la integran quienes no realizaron actividades económicas en la semana de referencia, ni buscaron hacerlo en los dos meses previos de la semana de referencia.
} 
El ingreso promedio mensual alude a los salarios mínimos promedios devengados al mes, el salario mínimo (SM) es la cantidad mínima mensual en pesos mexicanos que debe recibir la población ocupada por el desempeño de su trabajo. ${ }^{15}$ En promedio, las poblaciones retornadas analizadas ganan en general más de un salario mínimo diario al mes. Las proporciones más altas están en la categoría más de 3 SM —aproximadamente, MEX\$6309 al mes-. La población con mayor representación porcentual es la de mujeres del resto de retornados $(64,6 \%)$ y las mujeres retornadas indígenas $(55 \%)$. Las diferencias entre hombres y mujeres de estas poblaciones son de más de $10 \%$, lo cual evidencia una brecha amplia en salarios devengados según sexo.

En la tabla 4 se muestra la continuación de indicadores relativos al trabajo. En primer lugar, se detallan las prestaciones laborales que tienen la población ocupada. En términos generales, las mujeres retornadas muestran mayor presencia porcentual en todas las prestaciones analizadas respecto de los varones. La prestación con menores porcentajes es el reparto de utilidades y la que tiene el mayor porcentaje es el aguinaldo. Las prestaciones examinadas tienen una tendencia muy similar: las diferencias más relevantes son las de los hombres respecto a las mujeres.

Tabla 4. Población migrante de retorno, indicadores de resultados.

\begin{tabular}{|l|c|c|c|c|c|c|}
\hline & Hombres & Mujeres & $\begin{array}{c}\text { Hombres } \\
\text { Afro }\end{array}$ & $\begin{array}{c}\text { Mujeres } \\
\text { Afro }\end{array}$ & $\begin{array}{c}\text { Hombres } \\
\text { indígenas }\end{array}$ & $\begin{array}{c}\text { Mujeres } \\
\text { indígenas }\end{array}$ \\
\hline \multicolumn{7}{|c|}{ Prestaciones laborales de la población ocupada \% } \\
\hline Aguinaldo & 44,3 & 59,9 & 31,4 & 53,2 & 33,7 & 44,1 \\
\hline $\begin{array}{l}\text { Vacaciones con goce } \\
\text { de sueldo }\end{array}$ & 37,8 & 56,7 & 26,2 & 47,7 & 26,3 & 38,5 \\
\hline Servicio médico & 40,7 & 54,1 & 25,1 & 52,8 & 29,9 & 37,7 \\
\hline Reparto de utilidades & 26,2 & 34,2 & 16,7 & 38,9 & 17,7 & 20,7 \\
\hline Ahorro para el retiro & 32,4 & 45,7 & 20,7 & 49,7 & 22,2 & 26,8 \\
\hline Crédito para vivienda & 29,2 & 42,2 & 16,0 & 47,7 & 19,3 & 25,5 \\
\hline
\end{tabular}

\footnotetext{
${ }^{15}$ Se utiliza como escala de medición para indicar el ingreso por trabajo y su vigencia corresponde a la semana de referencia de la encuesta. Se tomó como base el salario mínimo mensual de la zona A, que correspondía a MEX\$2103, vigente al 31 de marzo de 2015 (Inegi, 2015).
} 
Tabla 4. (Continuación).

\begin{tabular}{|c|c|c|c|c|c|c|}
\hline & Hombres & Mujeres & $\begin{array}{c}\text { Hombres } \\
\text { Afro }\end{array}$ & $\begin{array}{l}\text { Mujeres } \\
\text { Afro }\end{array}$ & $\begin{array}{l}\text { Hombres } \\
\text { indígenas }\end{array}$ & $\begin{array}{l}\text { Mujeres } \\
\text { indígenas }\end{array}$ \\
\hline \multicolumn{7}{|c|}{ Relativos al derecho al trabajo } \\
\hline \multicolumn{7}{|c|}{ Actividades vinculadas con trabajo no remunerado \% } \\
\hline $\begin{array}{l}\text { Atender a personas } \\
\text { con discapacidad y } \\
\text { personas enfermas }\end{array}$ & 4,6 & 6,4 & 4,6 & 8,0 & 6,8 & 9,6 \\
\hline $\begin{array}{l}\text { Atender a personas } \\
\text { sanas menores } \\
\text { de } 15 \text { años }\end{array}$ & 29,7 & 56,7 & 33,5 & 58,8 & 37,0 & 68,0 \\
\hline $\begin{array}{l}\text { Atender a personas } \\
\text { de } 60 \text { y más años }\end{array}$ & 4,2 & 5,8 & 5,7 & 7,7 & 6,5 & 8,3 \\
\hline $\begin{array}{l}\text { Preparar o servir } \\
\text { alimentos para su } \\
\text { familia }\end{array}$ & 37,9 & 82,8 & 38,2 & 82,2 & 40,1 & 84,8 \\
\hline $\begin{array}{l}\text { Limpiar su casa, lavar } \\
\text { o planchar la ropa } \\
\text { de su familia }\end{array}$ & 42,7 & 86,4 & 45,0 & 85,2 & 44,2 & 88,8 \\
\hline $\begin{array}{l}\text { Hacer compras para } \\
\text { la comida o limpieza }\end{array}$ & 46,8 & 78,2 & 43,7 & 79,6 & 48,6 & 79,0 \\
\hline
\end{tabular}

Fuente: elaboración propia a partir de (Inegi, 2015).

El último indicador analizado es el trabajo no remunerado, el cual consiste en actividades realizadas por los integrantes de la vivienda sin recibir pago en beneficio propio por parte de los demás integrantes o incluso de personas de otras viviendas. La visibilidad de este indicador da la pauta para identificar y cuantificar las actividades de cuidado y atención de personas, entre otras, actividades que generalmente se asocian a las mujeres.

Es evidente que las actividades vinculadas al trabajo no remunerado de la población retornada siguen el mismo patrón que la población en general. Estas actividades, son predominantemente realizadas por las mujeres, sin importar su categorización. Sin embargo, las mujeres retornadas indígenas son quienes presentaron la mayor participación porcentual respecto al resto de las mujeres retornadas en todas las actividades analizadas.

En la mayoría de las actividades vinculadas al trabajo doméstico cocinar, limpiar, lavar y hacer compras- las diferencias entre sexos son 
amplias y las mujeres exhiben una preeminencia significativa en el desarrollo de estas. En actividades relacionadas con el cuidado de adultos mayores y personas enfermas los porcentajes muestran cierta paridad, empero, la carga de la actividad también recae mayoritariamente en términos porcentuales en las mujeres.

Los indicadores anteriores muestran un acercamiento a los derechos sociales de los que es poseedora la población retornada en el país y en los que se exhibe la influencia del género, la etnia y las características físicas en ciertos derechos y características específicas, panorama que da cuenta de la importancia del análisis interseccional y de su vinculación con los derechos humanos de la población migrante.

\section{Conclusiones}

El presente trabajo vincula, desde una perspectiva basada en los indicadores de derechos humanos establecidos por la OACDH (2012), las características, desigualdades y contrastes entre la población migrante de retorno procedente de Estados Unidos a México. El eje central son las mujeres retornadas como sujetos de análisis; sin embargo, se consideró que

[ 74 ] existen otras características que pueden incidir en el pleno desarrollo de los derechos sociales analizados, a saber: el género, la etnia y la consideración de afrodescendientes.

Los indicadores presentados muestran la estructura jurídica internacional y nacional, así como la política pública particular: la estrategia Somos Mexicanos, con la que el Gobierno abordó los principios e intereses en materia de derechos humanos para con la población migrante de retorno. A través de los indicadores de resultado derivados de la información proviene de la Encuesta Intercensal 2015 en México (Inegi, 2015), fue posible analizar con mayor profundidad la promoción y protección de los derechos sociales de las mujeres retornadas a territorio nacional.

Los datos mostrados ofrecen un panorama general en el cual el retorno femenino es minoritario en términos de volumen poblacional. La edad promedio de las retornadas oscila entre los 32 y 33 años, es decir, mujeres en edad activa para actividades laborales. Respecto a los derechos sociales analizados es posible mencionar que, sin distinguir etnia o afrodescendencia, las mujeres están en términos porcentuales mayoritariamente fuera del 
mercado laboral y tienen mayores actividades de trabajo no remunerado dentro de sus hogares. Lo anterior se acrecienta para mujeres indígenas retornadas y para las afrodescendientes. También de forma generalizada, las mujeres están en mayor grado registradas en otro país, el porcentaje respecto del total de las poblaciones analizadas es mínimo (entre 1 y $2 \%$ ), pero evidencia diferencias respecto a los hombres vinculadas al derecho a la identidad.

En cuanto a las características relacionadas con el derecho a la vivienda, se mostraron diferencias significativas entre las y los retornados, sobre todo respecto a la disponibilidad de servicios en la vivienda, a los que la población afrodescendiente tiene notablemente menor acceso.

Resulta importante destacar las características educativas de las mujeres retornadas, cualidades que las definen como un grupo heterogéneo. Son las que, porcentualmente, cuentan con mayor nivel educativo respecto a los hombres - nivel medio y superior-, pero también como grupo exhiben un porcentaje alto de mujeres indígenas con analfabetismo. Asimismo, las mujeres que son parte de la población que trabaja, obtuvieron mayores ingresos y mejores porcentajes de prestaciones laborales.

Lo anterior, vinculado a la legislación y la política pública, refuerza la justificación de realizar políticas de incidencia considerando características centrales como el género y la etnia. Es imprescindible abordar las particularidades específicas de la población. Las retornadas, como grupo de análisis de este trabajo, no son un ente homogéneo al que deba atenderse con un programa público específico. Es importante examinar otras categorías interseccionales con el fin de proveer un apoyo a medida y con impacto real. En este trabajo se devela que la población femenina, indígena y afrodescendiente apela a programas y políticas sectoriales pertinentes. Para que la migración de retorno pueda alcanzar su pleno potencial como posibilitadora del desarrollo es necesario concebirla y planificarla en el marco de los derechos humanos, tanto para los Estados como para los migrantes en su individualidad, como seres humanos.

Así, resulta preminente, tanto en México como en los países de Latinoamérica, establecer mecanismos de apoyo hacia los sectores más vulnerables e incidir en derechos sociales, reconociendo las desigualdades y diferencias. Es necesario que los Estados asuman el enfoque de igualdad en derechos con el fin de conformar sociedades más incluyentes. 


\section{Referencias bibliográficas}

1. ACNUR Comité Español. (2016, junio). ¿Cuáles son los derechos sociales y qué aplicación tienen? Agencia de la ONU para los Refugiados. https://eacnur.org/ blog/cuales-los-derechos-sociales-aplicacion-tienen/

2. Asamblea General de las Naciones Unidas. Resolución 2200 A (xxI). (3 de enero de 1976). Pacto Internacional de los Derechos Económicos, Sociales y Culturales. Oficina del Alto Comisionado de las Naciones Unidas para los Derechos Humanos. https://www.ohchr.org/sp/professionalinterest/pages/cescr.aspx

3. Asamblea General de las Naciones Unidas. Resolución 45/148. (18 de diciembre de 1990). Convención internacional sobre la protección de los derechos de todos los trabajadores migratorios y de sus familiares. Oficina del Alto Comisionado de las Naciones Unidas para los Derechos Humanos. https://www.ohchr.org/sp/ professionalinterest/pages/cmw.aspx

4. Calavita, Kitty. (2006). Gender, Migration, and Law: Crossing Borders and Bridging Disciplines. International Migration Review, 40 (1), pp. 104-132. https://doi. org/10.1111/j.1747-7379.2006.00005.x

5. Comité de las Naciones Unidas de Derechos Económicos, Sociales y Culturales (CESCR). E/C.12/GC/20. (2 de julio de 2009). Observación general N.o 20. La no discriminación y los derechos económicos, sociales y culturales del comité (art. 2, párr. 2 del Pacto Internacional de Derechos Económicos, Sociales y Culturales).

[ 76 ] https://www2.ohchr.org/english/bodies/cescr/docs/E.C.12.GC.20_sp.doc

6. Consejo de Europa. Protocolo número 4 al Convenio para la protección de los Derechos Humanos y de las Libertades Fundamentales. (16 de septiembre de 1963). https://www.derechoshumanos.net/Convenio-Europeo-de-Derechos-Humanos$\mathrm{CEDH}$ /1963-Protocolo04-ConvenioProteccionDerechosHumanosyLibertadesFunda mentales.htm

7. Consejo Nacional de Población (Conapo). (2017). Boletín de Migración Internacional. https://www.gob.mx/cms/uploads/attachment/file/213594/ Migraboletin.pdf

8. Corte Interamericana de Derechos Humanos. Serie C, N.o 149. (4 de julio de 2006). Caso Ximenes Lopes vs. Brasil. https://www.corteidh.or.cr/docs/casos/articulos/ Seriec_149_esp.pdf

9. Corte Interamericana de Derechos Humanos. Serie C, N. ${ }^{\circ}$ 282. (28 de agosto de 2014). Caso de personas dominicanas y haitianas expulsadas Vs. República Dominicana. https://corteidh.or.cr/docs/casos/articulos/seriec_282_esp.pdf

10. Departamento de Asuntos Económicos y Sociales de las Naciones Unidas (Undesa) (s. f.). Sustainable Development Goals. https://sustainabledevelopment. un.org/sdgs

11. Departamento de Seguridad Interna (DHS). (s. f.). ICE Statistics FY 2020. https://www.ice.gov/features/ERO-2020 
12. El Colegio de México y Comisión Nacional de Derechos Humanos. (2019). Migración de retorno y derechos sociales. Barreras a la integración. México, D. F.: El Colegio de México. https://migracionderetorno.colmex.mx/wp-content/ uploads/2019/06/COMPILADO_WEB.pdf

13. Gandini, Luciana; Lozano, Fernando y Gaspar, Selene. (2015). El retorno en el nuevo escenario de la migración entre México y Estados Unidos. México, D. F.: Consejo Nacional de Población. https://www.gob.mx/cms/uploads/attachment/ file/39174/ElRetornoEnelNuevoEscenariodeMigracion.pdf

14. García, Rodolfo y Del Valle, Rosa Elena. (2016). Migración de retorno y alternativas de reinserción. Hacia una política integral de desarrollo, migración y desarrollo humano. Huellas de la Migración, 1 (1), pp. 181-194.

15. Instituto Nacional de Estadística y Geografía (Inegi). (2015). Encuesta Intercensal 2015 [Conjunto de datos]. https://www.inegi.org.mx/programas/intercensal/2015/

16. Instituto Nacional de Migración (INM). (2016, diciembre 5). INM brinda apoyo integral a miles mexicanos repatriados de Estados Unidos. Boletín N. ${ }^{\circ}$ 34/16. https:// www.gob.mx/inm/prensa/inm-brinda-apoyo-integral-a-miles-mexicanos-repatriadosde-estados-unidos?idiom $=$ es

17. Instituto Nacional de Migración (INM). (s. f. a). Estrategia Somos Mexicanos. Gobierno de México. https://www.gob.mx/inm/acciones-y-programas/estrategiasomos-mexicanos

18. Instituto Nacional de Migración (INM). (s. f. b). ¿Qué es Somos Mexicanos? Gobierno de México. https:/www.gob.mx/cms/uploads/attachment/file/133316/ Infografi_a_Somos_Mex_FINAL.pdf

19. México. Cámara de Diputados de H Congreso de la Unión. Constitución Política de Los Estados Unidos Mexicanos. (5 de febrero de 1917). http://www. diputados.gob.mx/LeyesBiblio/pdf/1_280521.pdf

20. México. Cámara de Diputados de H Congreso de la Unión. Ley de Migración. (25 de mayo de 2011). https://www.acnur.org/fileadmin/Documentos/ BDL/2011/7525.pdf

21. México. Cámara de Diputados de H Congreso de la Unión. Ley General de Desarrollo Social. (20 de enero de 2004). http://www.diputados.gob.mx/LeyesBiblio/ ref/lgds/LGDS_orig_20ene04.pdf

22. México. Cámara de Diputados de H Congreso de la Unión. Ley General de Población. (7 de enero de 1974). http://www.diputados.gob.mx/LeyesBiblio/ pdf/140_120718.pdf

23. México. Gobierno de la República. Plan Nacional de Desarrollo 20132018. https://observatorioplanificacion.cepal.org/sites/default/files/plan/files/ MexicoPlanNacionaldeDesarrollo20132018.pdf

24. México. Gobierno de la República. Plan Nacional de Desarrollo 20192024. https://observatorioplanificacion.cepal.org/sites/default/files/plan/files/Plan \%20 Nacional\%20de\%20Desarrollo\%20de\%20M\%C3\%A9xico.pdf 
25. México. Instituto Nacional de Migración. Programa Especial de Migración (PEM) 2014-2018. https://www.gob.mx/cms/uploads/attachment/file/44579/Plan_ Especial_de_Migracion.pdf

26. México. Secretaría de Gobernación. Acuerdo por el que la Secretaría de Gobernación y la Secretaría de Relaciones Exteriores implementan la estrategia «Somos Mexicanos». (6 de julio de 2016). http://www.dof.gob.mx/nota_detalle.php?c odigo $=5443723 \&$ fecha $=06 / 07 / 2016$

27. Mora, Luis. (2002). Las fronteras de la vulnerabilidad: género, migración y derechos sexuales y reproductivos. CEPAL, Serie 33, pp. 34-37. https://repositorio.cepal. org/bitstream/handle/11362/6669/S0311794_es.pdf?sequence = 1\&isAllowed =y

28. Oficina del Alto Comisionado de las Naciones Unidas para los Derechos Humanos (OACDH). (2012). Indicadores de derechos humanos. Guía para la medición y la aplicación. https://www.ohchr.org/documents/publications/human_ rights_indicators_sp.pdf

29. Organización para la Unidad Africana. Carta Africana de Derechos Humanos y de los Pueblos (Carta de Banjul). (27 de julio de 1981). ACNUR. https://www.acnur. org/fileadmin/Documentos/BDL/2002/1297.pdf 